\title{
Slip Deformation of Crystal Grains in Semi-Circular Notched Thin Sheet Specimen of Pure Titanium during Tensile Test
}

\author{
Takashi Ishida $^{1 *}$, Naoya Tada ${ }^{1}$, Takeshi Uemori ${ }^{1}$, and Junji Sakamoto ${ }^{1}$ \\ ${ }^{1}$ Graduate School of Natural Science and Technology, Okayama University, 3-1-1 Tsushimanaka, Kita-ku, Okayama, Japan \\ *p42d61dg@s.okayama-u.ac.jp
}

Introduction. Pure titanium has been gathering much attention in the field of small medical devices. Since medical devices have been getting smaller and thinner, the accurate evaluation of mechanical properties is required for small-sized titanium components. In this paper, we performed tensile tests using a pure titanium thin sheet specimen with double edge semi-circular notches. The microscopic height change and slip deformation of grains were discussed.

Specimen and Tensile Test. Thin sheet specimen with a pair of semi-circular notches was used. The specimen was heat-treated, electropolished, and chemical etched before the test to observe the crystal grains on specimen surface. The tensile test was performed using a compact type tension-compression material testing machine. At each load step, height distribution was measured by a reflection-configured DHM R1100 (Lyncee Tec, Lausanne, Switzerland) featured with a very high vertical resolution less than $1[\mathrm{~nm}]$ in air condition.

Average Height of Grains. 69 small areas were set in 13 grains which are located near two semi-circular notches and the average height of each area, $h_{\mathrm{m}}$, defined by

$$
h_{\mathrm{m}}=\frac{1}{n^{2}} \sum_{i=1}^{n} \sum_{j=1}^{n} h\left(x_{i}, y_{j}\right)
$$

was evaluated, where $h\left(x_{i}, y_{j}\right)$ is the height at $\left(x_{i}, y_{j}\right)$ and $n$ is the number of pixels. The size of evaluation area was $51 \times 51$ [pixel] $(1$ [pixel] $=0.148[\mu \mathrm{m}])$.

Slip Activity of Grains. Since multiple slip lines were observed on the surface of grains after the tensile test, main mechanism of plastic deformation is considered to be slip. In this study, the slip activation was evaluated based on the (1) Schmidt factor SF, (2) modified Schmidt factor MSF, which is defined as SF divided by the critical resolved shear stress (CRSS), and (3) slip activity $S A$ which is newly defined as the modified Schmidt factor taking the local stress concentration into consideration. CRSSs for basal, prismatic, and pyramidal slip systems of pure titanium were reported as 49, 37, and 197 [MPa], respectively ${ }^{(1)}$. The values of MSF and SA were evaluated using these values. The local stress which is necessary to evaluate the value of $S A$ was calculated based on the theoretical stress distribution around a circular notch ${ }^{(2)}$.

Change of Average Height. The change in the average height with loading steps is shown in Fig. 1. Although the changes are small until the 7th step (elastic region), they become larger after that (plastic region), and it is also found that the changes are almost monotonic increase or decrease. Strong correlation between the height changes in elastic region and those in plastic region is also confirmed.

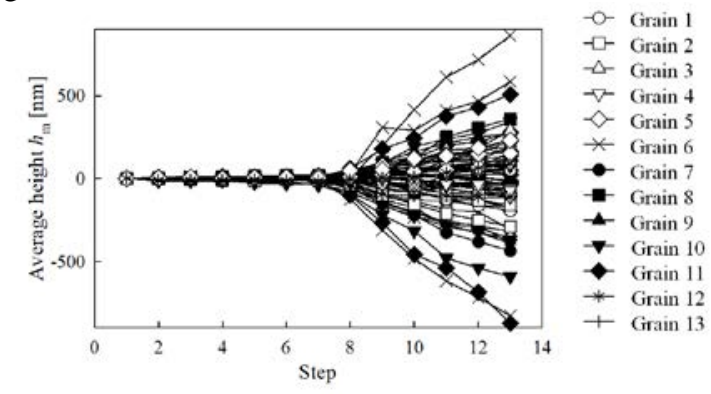

Fig. 1 Changes in the average height of grains with loading steps.

Prediction of Activated Slip Systems. Activated slip systems were predicted based on three parameters, $S F$, $M S F$, and $S A$. In this study, the prediction is considered to be correct when the angle of slip lines observed on the specimen surface is within \pm 5 [deg] of the predicted value. The results are shown in Fig. 2. SA shows the highest rate among three parameters. This fact suggests that the consideration of localized stress concentration is necessary to predict the slip activation.

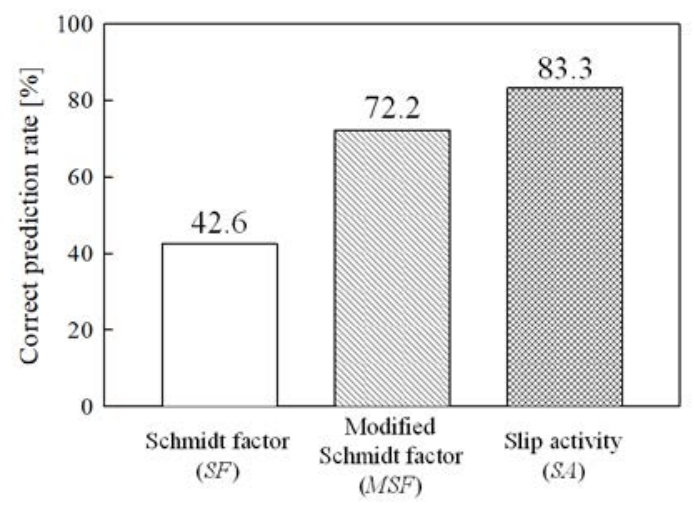

Fig. 2 Correct prediction rates for Schmidt factor, modified Schmidt factor, and slip activity.

\section{References.}

(1) Salem, A. A., Kalidindi, S. R. and Semiatin, S. L., Acta Materialia, Vol.53, (2005), 3495-3502.

(2) Timoshenko, S. P. and Goodier, J. N., Theory of Elasticity, 3rd ed., (1970), McGraw-Hill Int. Book, 78-82.

Acknowledgment. This research was supported by JSPS KAKENHI Grant Number JP18H01337. 